\title{
Identification of a Novel Salt-Tolerant Streptomyces Isolate with
}

\section{Bio-Fertilizing Property}

\author{
Rebah N. Algafari ${ }^{1 *}$, Ibrahim I. Hasan ${ }^{1}$ \& Ghayda A. Al-Joubory ${ }^{1}$ \\ ${ }^{1}$ Biotechnology Research Center, Al-Nahrain University, Baghdad, Iraq \\ *Rebah N. Algafari, E-mail: rebahalgafari@gmail.com
}

Received: August 11, 2016

Accepted: August 23, 2016 Online Published: September 1, 2016

doi:10.22158/se.v1n2p116

URL: http://dx.doi.org/10.22158/se.v1n2p116

\begin{abstract}
A 65 local Streptomyces isolates were tested for their salt tolerance ability. Four of them were found to grow on $6 \%$ salt concentration medium. These were selected as candidates for bio-fertilizing use. Only one of them named Streptomyces NS-38 was found to pose such trait by enhancing accelerated seed germination of different types of plants on salt and normal media. Result showed that incubation of seeds with this bacterium extract for 15 hours before implantation increased the number of germinating seeds and yield significantly.
\end{abstract}

\section{Keywords}

streptomyces, bio-fertilizers, halotolerant bacteria, bacterial IAA, cellulase

\section{Introduction}

Streptomyces genus, make an important group of soil bacteria from the actinobacteria class. Several species of the Streptomycetaceae family are widely studied because of their ample capacity for production of secondary metabolites, such as antibiotics and extracellular enzymes (Inbar et al., 2005). These microorganisms are abundant in soils and act in the degradation of complex molecules as well as recalcitrant substances, especially cellulose, lignocellulose, xylan and lignin, that play an important role in soil organic matter decomposition processes (Sousa et al., 2008).

These bacteria can be isolated mostly from all habitats, garden soils, hydrocarbon contaminated soil, saline soil, farm soil, and animal waste (Algafari, 2014).

The salinity in soils became an increasing problem, and use of chemical fertilizers expanded this problem to include irrigation water and rivers. Salinity is one major limiting factor to plant growth and crop productivity (Allakhverdiev et al., 2000). Cultivated soils worldwide are becoming more saline from marginal irrigation water, excessive fertilization, and desertification processes. Strategies for alleviation of salt stress involve developing salt-resistant cultivars, leaching excess soluble salts from upper to lower soil depths, flushing soils that contain soil crusts at the surface, reducing salt by 
harvesting salt-accumulating aerial plant parts in areas with negligible irrigation water or rainfall for leaching, and amelioration of saline soils under cropping and leaching (Bacilio et al., 2004). An alternative is to alleviate salt stress by inoculating crop seeds and seedlings with Plant Growth Promoting Bacteria (PGPB). Looking into the perspectives of crop production losses due to the severity of abiotic stresses, especially salinity, tolerance to stress provided by microbial inoculants becomes more important (Dhanushkodi et al., 2013).

The use of non-pathogenic bacteria to serve as bio-fertilizers seems to be the right choice, and streptomycetes are the perfect candidate since they are soil bacteria, and pose no disease to human, plants and animals.

\section{Materials and Methods}

\subsection{Bacterial Strains}

All bacterial strains tested were a part of Biotechnology Research center at Al-Nahrain University collection and they were isolated and identified by Algafari 2014.

\subsection{Selection of Salt Tolerant Strains}

Streptomyces under study were grown on ISP4 medium with salt concentration of $1 \%$ to $6 \%$ with increase of $1 \%$. Only those were able to grow on the higher concentration were selected for further study.

\subsection{Test for Bio-Fertilization}

Strain with salt - tolerance was grown on $2 \%$ salt concentration ISP4, and seeds from different plants were incubated with them and observed for germination. Bacterial strain activated seeds germination rapidly comparing to the control was selected in this study.

\subsection{Detection of Indole and Indol Acetic Acid (IAA)}

Detection of indole was made depending on the reaction with Salkowski's reagent, while detection of IAA was made according to the method of Narayana et at. (2009). The Streptomyces strain NS-38 was cultivated in YMD broth for 5, 7 and 10 days to perform this test.

\section{Results}

Streptomycetes are highly reliable soil bacteria, and they are an important element in eco-balance system since they pose major activities in this process like organic material decomposition, pathogenic fungi antagonism, and preserving micro-environment around plants roots. Moreover, involvement of this genus in bio-fertilization especially in salt soils is an important criteria needs to be investigated.

\subsection{Selection of Bio-Fertilizer}

Different seeds from wheat, tomato, and Lepidium sativum were grown with salt tolerant streptomycetes on $2 \%$ salt containing ISP4 medium and observed for germination. Only one strain named NS-38 was able to activate growth of the seeds and formation of the shoot as shown in Figure 1. 


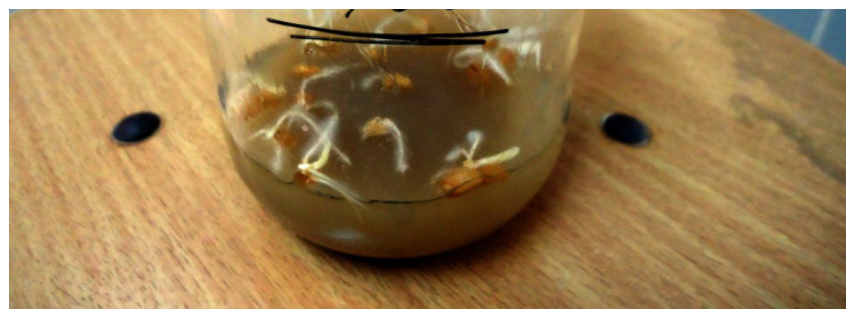

Figure 1. Germination of Wheat Seeds Grown in 2\% Salt Medium Incubated with N-38

\section{Streptomyces Strain}

This strain was found to have significant effect on seeds germination since about $95 \%$ of seeds were able to form roots and shoots comparing with control grown on the same medium without NS-38 as shown on Figure 2.

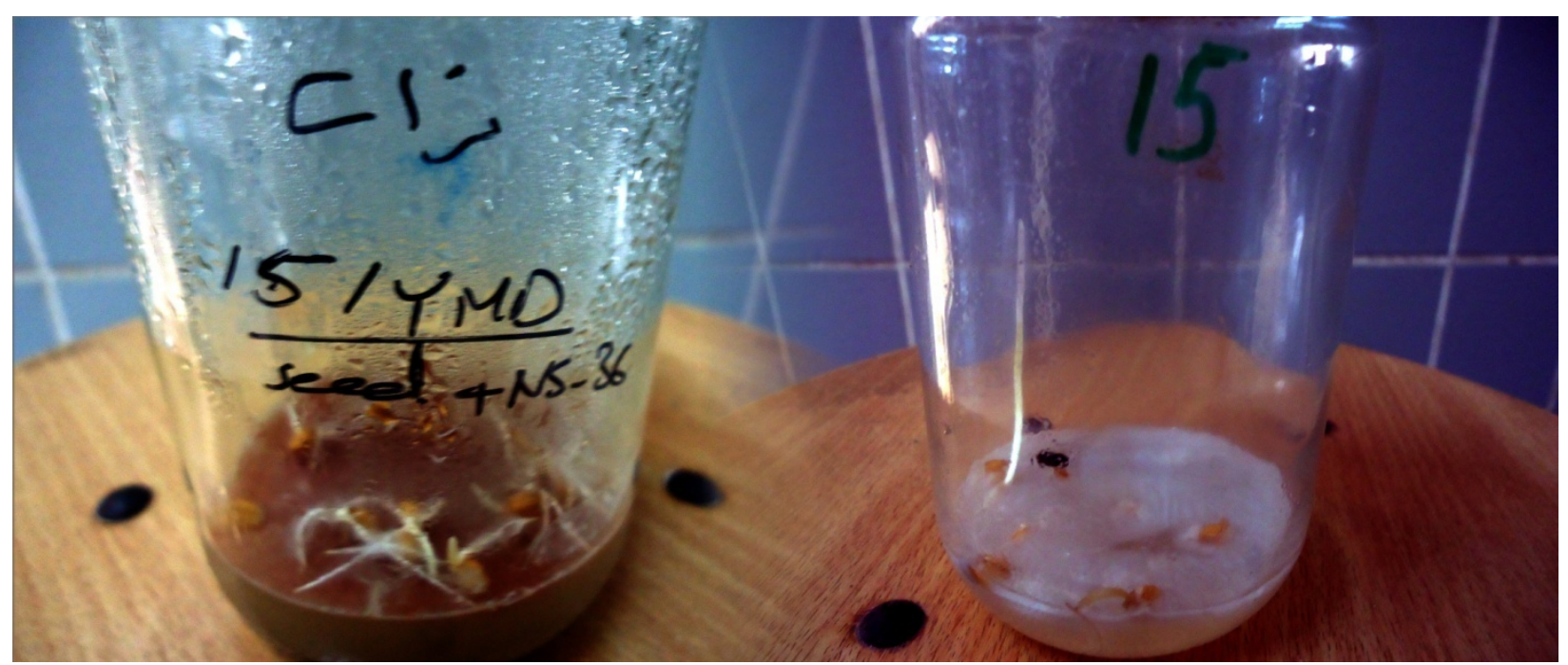

Figure 2. Wheat Seeds Germination

The tube to the left shows the germination and shoot formation to about $95 \%$ of the seeds, while the tube to the right shows low rate germination in the absence of NS-38. Both seeds were grown on MS medium with $2 \%$ salt concentration.

Some plants like tomato require long time to germinate when cultivated on MS medium. The extract obtained from NS-38 seemed to accelerate germination and shoot formation. The normal time to produce shoots from tomato seeds is two weeks, while when extract from NS-38 was used germination began in the 3rd day, and shoot formation began at the first week as shown in Figure 3. 


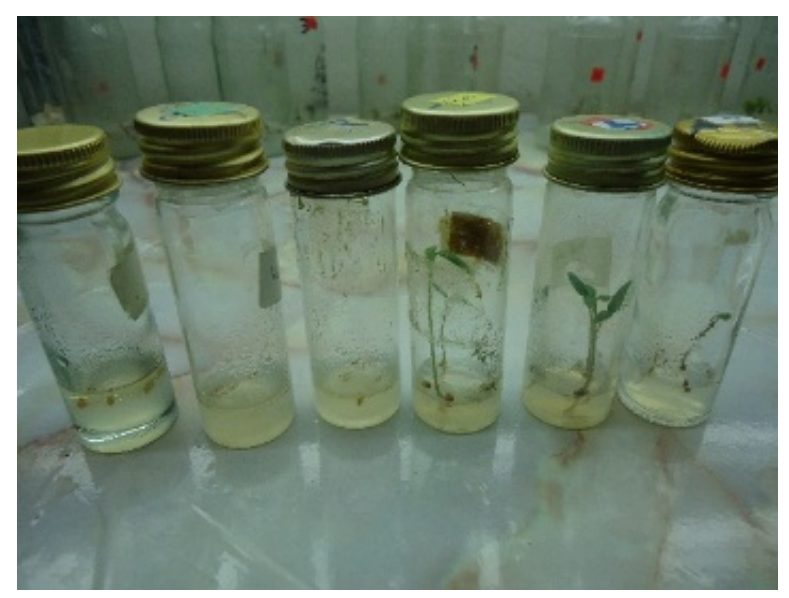

Figure 3. Germination and Shoot Formation of Tomato Seeds Cultivated on MS Medium

The three tubes to the left are control (seeds without NS-38 extract), and the three tubes to the right are tomato seeds treated with NS-38 extract. The Figure shows significant germination and shoots formation in seeds treated with microbial extract.

\subsection{Detection of Indole Production}

Streptomyces NS-38 was tested for indole production using Salkowski's reagent. The production of indole began since the 5 th day of cultivating the bacterium on ISP4 medium as shown in Figure 4.

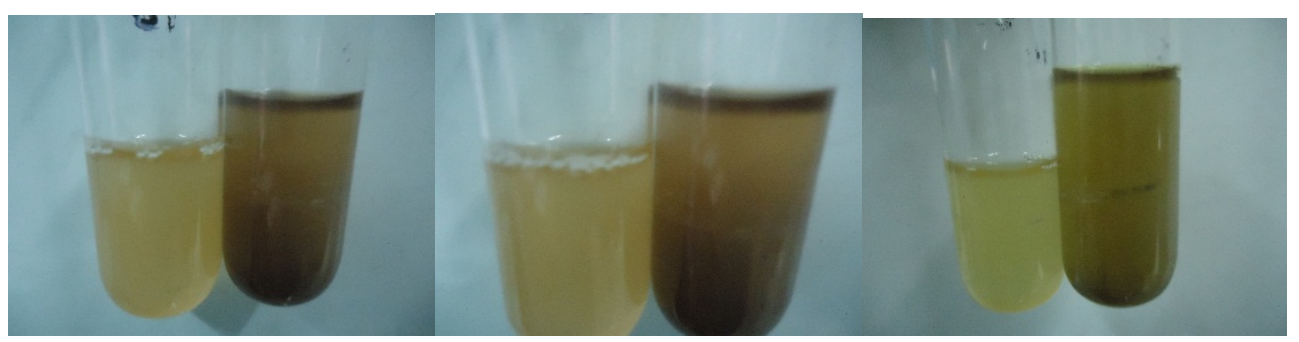

Figure 4. Indole Production by NS-38 Strain

The dark tube is the reaction of indole to Salkowski's reagent. No. 1 is the test after 5 days, no. 2 is the test after 7 days, and no. 3 is the test after 10 days of incubation in YMD medium.

Production of IAA was confirmed in the medium following the method of Narayana et at. (2009).

\subsection{Optimization of Seeds Incubation Period}

\subsubsection{Method of Treatment}

Two methods were used to expose seeds to the effect of NS-38 extract, first implanting seeds on MS medium and addition of the bio-fertilizer bacterium extract, second incubating seeds with the bio-fertilizer, and then implanting them on MS medium. We found that incubating seeds with bio-fertilizer extract made the maximum effect on seeds germination. 


\subsubsection{Time of Incubation}

Seeds germinated were incubated with bio-fertilizer extract for different period of time started from 1 hour to 24 hour with 1 hour increase of time. The critical time needed to get maximum germination of seeds before forming roots and shoots during incubation was 18 hours as shown in Figure 5.

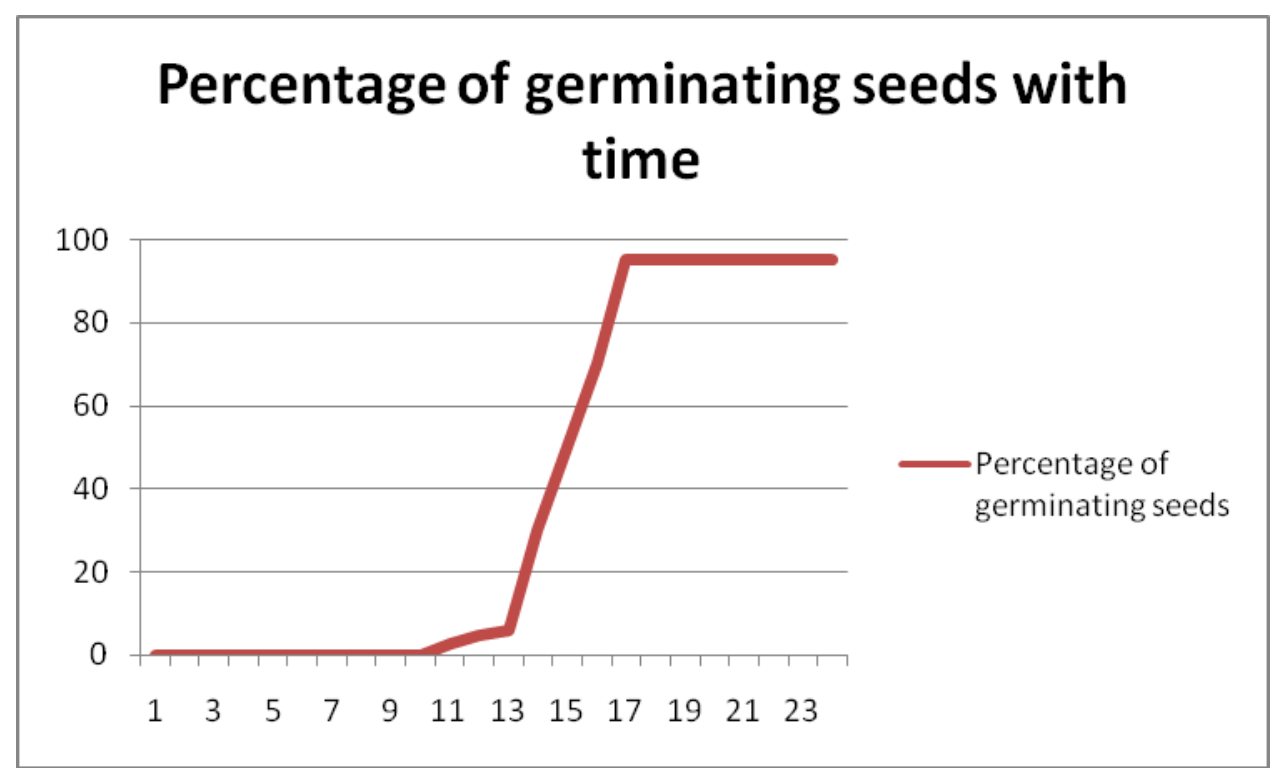

Figure 5. Percentage of Germinating Seeds with Time

The $\mathrm{Y}$ axis represents the percentage of germinating seeds, and the $\mathrm{X}$ axis is time in hours.

An important note we came on during this work is that the extract from NS-38 activated seed germination from Lepidium sativum at low ambient temperature of $10 \mathrm{C}$ rapidly and produced full plant in 1 week of cultivation in soil as shown in Figure 6.

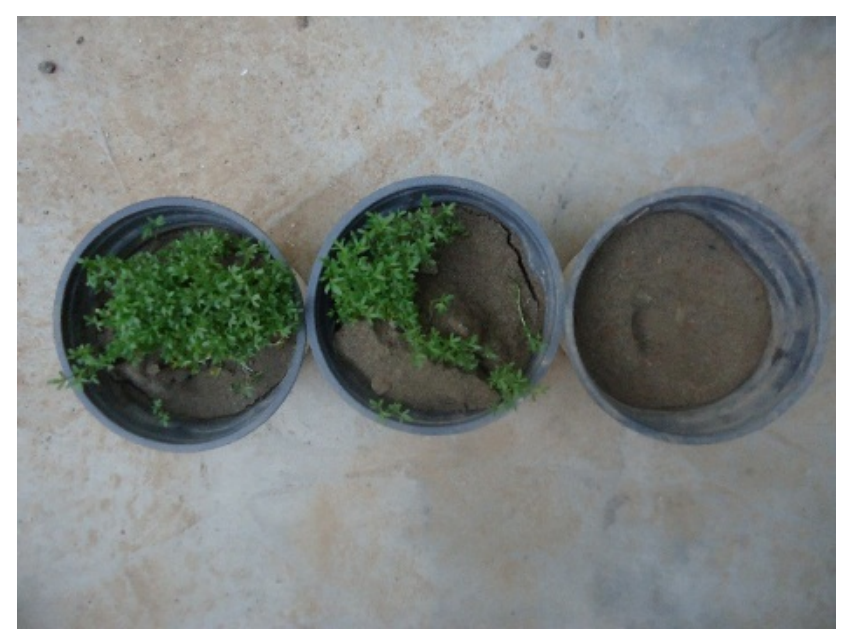

Figure 6. Cultivation of Lepidium Sativum in Soil at Low Ambient Temperature 
The two pots to the left are NS-38 extract treated seeds, the pot to the right is control. The photo was taken after 1 week of cultivation.

\section{Discussion}

Streptomycetes are very important bacteria in the nature. They can preserve soil ecosystem significantly by participating in the decomposition of organic matter and production of secondary metabolites that involve in the antagonism of other living organisms and providing protection to plants against pathogens like fungi and worms. In many cases such secondary metabolites may be plant hormones or plant hormones analogous like IAA. These may aid seeds germination and increase yield of crops. The bacterium NS-38 studied in this article was found to produce cellulase and able to decompose cellulose added to the cultivation plate significantly (unpublished data). In addition, production of IAA in the medium was another factor that aided seeds germination rapidly and efficiently and caused to produce complete plant in less time and higher yield. The effect of salt in the medium was diluted when the bacterium extract was added and almost all seeds were able to germinate and produce complete plant suggesting the presence of other mechanism that lower the stress of salt on seeds and growing plant.

\section{References}

Al-Gafari, R. N. (2014). Assessment of profile depth, site of sampling, type of media and methods used for the isolation of actinomycetes. International Journal of Microbiology Research, 6, 553-558.

Allakhverdiev, S. I., Sakamoto, A., Nishiyama, Y., Inaba, M., \& Murata, N. (2000). Ionic and osmotic effects of NaCl-induced inactivation of Photosystems I and II in Synechococcus sp. Plant Physiol, 123, 1047-1056. http://dx.doi.org/10.1104/pp.123.3.1047

Bacilio, M., Rodriguez, H., Moreno, M., Hernandez, J. P., \& Bashan, Y. (2004). Mitigation ofn salt stress in wheat seedlings by a gfp-tagged Azospirillum lipoferum. Biol Fertil Soils, 40, 188-193. http://dx.doi.org/10.1007/s00374-004-0757-z

Carla da Silva Sousa Ana Cristina Fermino Soares, \& Marlon da Silva Garrido. (2008). Characterization of Streptomycetes with potential to promote plant growth and biocontrol. Sci. Agric, 65, 50-55. http://dx.doi.org/10.1590/S0103-90162008000100007

Dhanushkodi, R., Vithal, K. L., Pranita, B., Sajad, A., \& Kannepalli, A. (2013). Mitigation of salt stress in wheat seedlings by halotolerant bacteria isolated from saline habitats. Springer plus, 2, 1-7.

Inbar, E., Green, S. J., Hadary, Y., \& Minz, D. (2005). Competing factors of compost concentration and proximity to root affect the distribution of streptomycetes. Microbiology Ecology, 50, 73-81. http://dx.doi.org/10.1007/s00248-004-0111-x

Narayana, K. J., Prabhakar, P., Venketewarlu, E., \& Vijayalakshmi, M. (2009). Indole-3-acetic acid production by Streptomyces albidoflavus. Journal of Biological Research-Thessaloniki, 11, 49-55. 